\title{
Maltrato al adulto mayor, un problema silencioso
}

\author{
Elder abuse, a silent problem
}

\author{
Maus-tratos aos idosos, um problema silencioso
}

\author{
Maite Catalina Agudelo-Cifuentes', Doris Cardona-Arango², Ángela Segura-Cardona33, Diego Alveiro Restrepo-Ochoa4. \\ 1 Estudiante de Doctorado en Epidemiología y Bioestadística. Universidad CES, Medellín-Colombia. magudeloc@ces.edu.co. ORCID: \\ orcid.org/0000-0003-1501-9452. \\ 2 Docente investigadora. Universidad cEs, Medellín-Colombia. dcardona@ces.edu.co. ORCID: orcid.org/0000-0003-4338-588X. \\ 3 Directora Escuela de Graduados. Universidad cEs. Medellín-Colombia. asegura@ces.edu.co. orcid: orcid.org/0000-0002-0010-1413. \\ 4 Decano Facultad de Psicología. Universidad cEs, Medellín-Colombia. drestrepo@ces.edu.co. orcid: orcid.org/0000-0002-4469-6260.
}

Recibido: 17/02/2018. Aprobado: 17/10/2019. Publicado: 20/03/2020

Agudelo-Cifuentes MC, Cardona-Arango D, Segura-Cardona Á, Restrepo-Ochoa DA. Maltrato al adulto mayor, un problema silencioso. Rev. Fac. Nal. Salud Pública. 2020;38(2):e331289. DoI: https://doi.org/10.17533/udea.rfnsp.e331289

\section{Resumen}

Los malos tratos hacia las personas mayores están influenciados por patrones culturales, el contexto histórico, el entorno sociocultural y los juicios de valor que la sociedad construye en torno a la vejez. El maltrato a esta población no ha tenido la misma resonancia en la sociedad que el ejercido a niños y mujeres; sin embargo, en los últimos años ha comenzado a ser visible como un problema de interés público. La presente revisión tiene como objetivo presentar los principales conceptos y características del maltrato al adulto mayor y describir la magnitud de este fenómeno social en el mundo y en Colombia desde 1975 hasta 2018.

---------Palabras clave: adulto mayor, maltrato al adulto mayor, maltrato intrafamiliar, envejecimiento, Colombia.

\section{Abstract}

Abuse towards older people is influenced by cultural patterns, the historical context, the socio-cultural environment and the value judgments that society builds around old age. The mistreatment of this population has not had the same resonance in society as the abuse of children and women; however, in recent years it has started to be seen as a problem of public interest. This review aims to present the main concepts and characteristics of elder abuse and describe the magnitude of this social phenomenon worldwide and in Colombia from 1975 until 2018.

---------Key words: elder, elder abuse, domestic violence, aging, Colombia. 


\section{Resumo}

Os maus-tratos aos idosos estão influenciados por padrões culturais, o contexto histórico, o ambiente sociocultural e os juízos de valor que a sociedade constrói ao redor da velhice. Os maus-tratos dirigidos a esta população não têm tido a mesma repercussão na sociedade que o exercido às crianças $\mathrm{e}$ mulheres; porém, nos últimos anos se tornou mais visível como um problema de interesse público. A presente revisão tem como objetivo apresentar os principais conceitos e características dos maus-tratos ao idoso e descrever a extensão desse fenômeno social na Colômbia e no mundo desde 1975 até 2018.

-Palavras-chave: idoso; maus-tratos ao idoso; maustratos intrafamiliar; envelhecimento; Colômbia

\section{Introducción}

El envejecimiento poblacional representa un logro de la salud pública que refleja el éxito de las políticas en salud y desarrollo económico [1]. La reducción en la mortalidad infantil y las mejoras en atención de la salud han permitido que las personas puedan vivir más años; sin embargo, para muchos adultos mayores, los años de vida adicionales no son vividos con calidad [2].

La etapa de la vejez ha sido asumida culturalmente como sinónimo de fragilidad y dependencia, lo cual configura un conjunto de ideas negativas frente al envejecimiento, que puede llevar a discriminación, aislamiento social y maltrato [3].

El maltrato hacia la población mayor se presenta a nivel mundial [4]. Aunque las cifras pueden ser más altas en quienes habitan hogares de ancianos que en los que se encuentran en la comunidad, el maltrato al interior de los hogares es cada vez mayor [4]. Este problema es altamente subestimado, principalmente por el miedo de las víctimas a reportarlo [5], lo que, sumado a la ausencia de instrumentos adecuados para su detección, contribuye a que continúe oculto [1].

Los malos tratos hacia las personas mayores están influenciados por patrones culturales, el contexto histórico, el entorno sociocultural y por el valor que se da a la vejez. Este tipo de violencia no ha tenido históricamente el mismo eco que el ejercido a niños y mujeres; sin embargo, en los últimos años ha comenzado a ser tema de interés público [6, p. 541].

Colombia es un país que se encuentra en transición demográfica, influenciada por la disminución de la mortalidad y de la fecundidad. La población mayor ha aumentado a un ritmo superior al $3 \%$ anual. En 1950, la esperanza promedio de vida al nacer era de 48 años, aumentando para el 2010 a 74 años, siendo mayor el incremento para la población femenina [7]. En el país, la vejez se acompaña de escasa seguridad económica y falta de cobertura en pensiones, principalmente en las mujeres [8], en quienes el envejecimiento llega por lo general acompañado de soledad, lo que aumenta la vulnerabilidad social y financiera, principalmente por el bajo nivel educativo y la dependencia económica [9]. Se estima que el $50 \%$ de la población mayor en Colombia tiene estudios primarios, el $20 \%$ es analfabeta y cerca del $30 \%$ realiza trabajo de manera informal [8].

El maltrato hacia los adultos mayores en el país ha sido un tema poco explorado; sin embargo, algunas aproximaciones reflejan una alarmante situación $[8,10$ 12], la cual debe ser visibilizada y estudiada, dado que este problema afecta en diferente forma a todas las sociedades. Por esto, el presente ensayo tiene como objetivo presentar los principales conceptos y características del maltrato al adulto mayor y describir la magnitud de este fenómeno social en el mundo y en Colombia desde 1975 hasta 2018.

\section{Aproximaciones teóricas al concepto de maltrato al adulto mayor}

En este apartado se hace un recorrido por las principales concepciones que han surgido sobre el maltrato al adulto mayor desde 1975 hasta 2018.

Para elaño 1975 se comienza a considerar teóricamente la población adulta mayor como vulnerable a maltrato, por medio de una carta publicada en la revista The Bristinsh Medical Journal, titulada "Granny-battering" [13], en la cual destaca que el maltrato hacia la población mayor es un problema existente que suele permanecer en silencio, enmascarado en caídas u otras situaciones que justifican lo que pudiera ser un mal cuidado por parte de los familiares. Hacia 1986, Johnson [14] define estos hechos como cualquier sufrimiento físico o emocional al que se ve sometido una persona mayor y que afecta de alguna manera su calidad de vida.

Alrededor de 1991, Hudson, publicó un artículo titulado: "Elder mistreatment: A taxonomy with definitions by Delphi", en el que define este problema como "una conducta destructiva que está dirigida a una persona mayor, ocurre en el contexto de una relación que denota confianza, y reviste suficiente intensidad o frecuencia para producir efectos nocivos de carácter físico, psicológico, social o financiero, de innecesario sufrimiento, lesión, dolor, pérdida o violación de los derechos humanos y disminución en la calidad de vida" [15].*

* Las traducciones a lo largo del artículo fueron realizadas por Maite Catalina Agudelo Cifuentes. 
Para el año 1996 fue desarrollada la "Declaración de Almería sobre el Anciano Maltratado", la cual surgió en el marco de la "Primera Conferencia Nacional de Consenso sobre el Anciano Maltratado". En esta declaración se define el maltrato hacia personas mayores como "cualquier acto u omisión que produzca daño, intencionado o no, practicado sobre personas de $65 \mathrm{y}$ más años, que ocurra en el medio familiar, comunitario o institucional, que vulnere o ponga en peligro la integridad física, psíquica, así como el principio de autonomía o el resto de los derechos fundamentales del individuo, constatable objetivamente o percibido subjetivamente" $[16$, p. 1]

En Colombia, para 1997, en el Foro Nacional sobre Tercera Edad, realizado en Bogotá, Álvarez [17] habla acerca de la marginación y el maltrato al adulto mayor, donde lo define como cualquier acción voluntaria, accidental o fortuita, que lleva a ofensa o descuido de una persona mayor de 60 años, por algún familiar, por un tercero o por la sociedad.

Por otro lado, la Organización Panamericana de la Salud, en su "Informe mundial sobre la violencia y la salud" de 2002, indica que el maltrato es una cuestión de apreciación, por lo cual su definición no es exacta y puede ser difusa y compleja, dado que identificar un hecho o un comportamiento como inaceptable o dañino, tiene una alta carga cultural [18].

En el año 2003, la Organización Mundial de la Salud elaboró la "Declaración de Toronto para la prevención global del maltrato de las personas mayores" [19], documento en el que la definición de este problema se asemeja un poco a las anteriores mencionadas, pero atañen estos hechos a personas con quien el adulto mayor comparte algún vínculo. En este sentido, la declaración define el maltrato como cualquier acción u omisión que se presenta de forma única o repetida, que causa daño o angustia al adulto mayor, y que se genera en una relación en la que existe algún grado de confianza, dependencia o expectativa.

En 2004, Muñoz, en su libro Personas mayores $y$ malos tratos [20, pp. 15-37], sugiere una definición similar a las ya conocidas hasta el momento, haciendo mayor énfasis en el efecto que tienen estos hechos sobre la calidad de vida de la víctima. Así, su definición engloba también cualquier acto u omisión hacia una persona adulta mayor, que se genere ya sea en el entorno familiar o institucional, que atenta contra su vida, seguridad, integridad física, psicológica o que comprometa su calidad de vida. Además, Muñoz indica que este problema se potencializa con las relaciones jerarquizadas que envuelven la sociedad, siendo el maltrato a esta población una de las últimas formas de violencia identificada como problema social.

Han surgido otras definiciones similares, como la de De Paúl y Larrión, en el año 2006 [21], en su texto "El maltrato a los mayores. Algunas cuestiones generales", donde lo definen como situaciones de malestar físico, de privación o psicológico, como resultado de un acto u omisión de quien se encarga del cuidado. O la definición dada en 2008 por Iborra Marmolejo, en su libro Maltrato de personas mayores en la familia en España: "cualquier acción voluntariamente realizada, es decir, no accidental, que dañe o pueda dañar a una persona mayor; o cualquier omisión que prive a un adulto mayor de la atención necesaria para su bienestar, así como cualquier violación de sus derechos" [22, p. 14]. En esta última, la autora hace un especial énfasis en la intencionalidad de los hechos, al acuñar el maltrato a situaciones netamente accidentales.

En Chile, para el año 2012, el Servicio Nacional del Adulto Mayor publicó la Guía Prevención del maltrato a las personas mayores, en la cual se define este problema como "cualquier acción u omisión que produce daño y que vulnera el respeto a su dignidad y el ejercicio de sus derechos como persona" [23]. Asimismo, en México, para el año 2013, en la Guía de práctica clínica. Detección y manejo del maltrato en el adulto mayor se define este problema como "cualquier acto u omisión que tenga como resultado un daño, que vulnere o ponga en peligro la integridad física o psíquica, así como el principio de autonomía y respeto de sus [sic] derechos fundamentales del individuo de 60 años o más, el cual puede ocurrir en el medio familiar, comunitario o institucional" [24, p. 11].

Las distintas definiciones existentes sobre esta problemática coinciden en que el maltrato no es únicamente una acción, sino que, por el contrario, la falta de cuidados necesarios a personas de mayor edad también se constituye como maltrato. Así mismo, se observan diferencias entre estas, enmarcadas principalmente en la intencionalidad o no de causar daño y en el vínculo existente entre victimario y víctima. Sin lugar a duda, todas estas definiciones dejan de manifiesto que estos hechos vulneran y atentan contra el bienestar de la persona mayor en todos los ámbitos.

\section{Tipología del maltrato al adulto mayor}

El maltrato hacia las personas mayores puede presentarse en diferentes formas [20], y con frecuencia, un adulto mayor maltratado es víctima de más de una forma de abuso $[25,26]$. Estos hechos han sido clasificados comúnmente en cinco tipos: físico, psicológico, económico, sexual y negligencia $[20,22]$. El Centro para el Control y Prevención de Enfermedades identifica estas cinco categorías y adiciona el abandono como forma de maltrato [5].

El maltrato físico es el uso intencional de la fuerza que termina en lesión corporal, dolor, deterioro 
funcional, enfermedad, angustia o muerte; es todo acto que genere lesiones o trastornos, y que incluye golpear, patear, quemar, empujar $[5,20,27]$. Este tipo de maltrato puede ser evidente por las marcas o lesiones que deja al adulto mayor, las cuales pueden ser visibles.

Caso contrario al anterior, el maltrato psicológico podría ser uno de los más subestimados y ocultos. Es cualquier agresión verbal o no verbal que atenta contra la dignidad de la persona y desencadena angustia o miedo [28], incluyendo humillaciones, amenazas, aislamiento, y control o limitación de determinadas actividades, como hablar por teléfono o salir de casa [5].

El maltrato económico o financiero es el uso ilegal, no autorizado o inapropiado de los recursos o propiedades del adulto mayor, en medio de una relación de confianza $y$ en pro del beneficio de otra persona [5,22].

Por otro lado, los adultos mayores también pueden ser víctimas de maltrato sexual, el cual incluye toda interacción sexual o contacto físico, directo o a través de la ropa, que no es deseado por la víctima y se ejerce bajo presión. Este tipo de maltrato atenta contra el pudor del adulto mayor y usualmente se mantiene en secreto $[5,20]$.

Otro tipo de maltrato, quizá más frecuente de lo que se estima, es la negligencia, definida esta como la falta de satisfacción de las necesidades de atención al adulto mayor, por parte de la persona responsable de su protección, que puede poner en riesgo la seguridad y la salud del adulto mayor [5,22]. Algunas de las manifestaciones de este tipo de maltrato son: la falta de atención médica esencial, nutricional, de hidratación, higiene, ropa y refugio, distanciamiento físico y descuido en el cuidado [22]. Por su parte, el autoabandono es la falta de autocuidado, o cualquier conducta que puede adoptar una persona, que amenaza su salud o seguridad, y que se debe a un rechazo hacia sí mismo [28].

Las prevalencias de maltrato pueden variar según los tipos [4]. El maltrato más frecuente en población mayor es el psicológico, seguido del económico, la negligencia, el físico y, en último lugar, el maltrato sexual $[29,30]$. Sin embargo, la frecuencia con la que se presenta cada tipo de maltrato varía de acuerdo con el contexto, el género $\mathrm{y}$ las condiciones socioeconómicas. El maltrato físico y sexual se presentan principalmente en mujeres [31], y la negligencia, principalmente en quienes tienen bajos ingresos y poco apoyo social [32].

\section{Características asociadas}

El maltrato al adulto mayor surge como resultado de la interacción de múltiples factores [27], entre los cuales están las características propias de la persona y las características familiares y sociales [33,34].
En cuanto a características propias de la persona, se ha encontrado que las principales víctimas de maltrato físico y sexual son las mujeres [12,31,35,36]; esto, debido, entre otras causas, a que la mujer informa más este tipo de situaciones, mientras que en los hombres suelen permanecer ocultas [36].

A partir de los 75 años, el riesgo de maltrato puede ser mayor [23], dado que, con la edad, aumentan también la dependencia, el deterioro de la salud y el aislamiento social, lo que podría llevar a índices superiores de estrés en la familia y en los cuidadores [22,23,35]. La probabilidad de maltrato es alta en los adultos mayores con deterioro cognitivo y demencia, pero es en esta población donde hay mayor dificultad para detectarlo [23,37,38].

Estudios han encontrado que el consumo de alcohol por el adulto mayor es un factor relacionado con el maltrato, debido a que este puede generar trastornos cognitivos y de la memoria, lo que aumenta la vulnerabilidad; sin embargo, la relación igualmente puede ser inversa, dado que los adultos mayores que son maltratados pueden recurrir al alcohol como forma de afrontar la situación [39].

Otros factores asociados son la pobreza, las actitudes negativas hacia la vejez $[19,22,34]$ y algunas características familiares y de la persona encargada del cuidado, como: antecedentes de maltrato al interior del hogar, convivencia continua, disfuncionalidad familiar [23], consumo de alcohol [38], estrés o sobrecarga [23], dependencia económica [22,23], problemas psicológicos [20,23,37,40] y aislamiento social [23].

Dependiendo de las características de las personas con las que convive la persona mayor o de quien se encarga de su cuidado, el riesgo de maltrato puede incrementar [31]. Sin embargo, en muchos casos estas situaciones pueden no tener implícita una intención de daño, siendo el resultado del desconocimiento del daño de las propias acciones por parte de personas cercanas $[41,42$, p. 15$]$.

En situaciones de adversidad, incertidumbre o temor, el apoyo social actúa como una fuente de protección, tanto a nivel individual como familiar [43,44]; en este sentido, las relaciones sociales tienen influencia positiva en las relaciones afectivas al interior de las familias [44]. El Instituto Nacional de Medicina Legal y Ciencias Forenses de Colombia, en su publicación Violencia al adulto mayor en el contexto intrafamiliar, reportó que las deficiencias en las redes sociales del adulto mayor se convierten en un factor que facilita la presencia de maltrato a esta población [45], lo cual ha sido mencionado por otros autores, como Bover et al. [6].

Por otra parte, la imagen o concepto que la sociedad se forma de la vejez, la concepción social de esta como una etapa de fragilidad y dependencia, el debilitamiento de los vínculos familiares y la falta 
de recursos económicos para garantizar el adecuado cuidado de la población envejecida se constituyen en factores predisponentes para la presentación de este tipo de hechos violentos al interior de las familias [46].

En cuanto a las consecuencias o repercusiones que se pueden derivar de estos hechos se encuentran problemas de autoestima y aumento en la inseguridad, condiciones que propician la generación de estereotipos negativos del envejecimiento [47]. Además, tiene repercusiones físicas, psicológicas y sociales [48] y genera consecuencias negativas sobre la salud y la calidad de vida [24]. A nivel psicológico, puede causar tristeza, sufrimiento, trastornos emocionales $\mathrm{y}$ depresión [49]; los comportamientos o ideas suicidas, y sentimientos de infelicidad, vergüenza o culpabilidad [6,31] han mostrado ser comunes entre los adultos mayores víctimas de maltrato. Este problema, además, se ha encontrado asociado con el aumento de la morbilidad [50], hospitalización y muerte [50-52]. Quienes han sido víctima de maltrato son más propensos a tener mayor riesgo de mortalidad y menor esperanza de vida que aquellos que nunca han pasado por situaciones de violencia [50].

\section{Formas de medición del maltrato}

El maltrato a la población adulta mayor, aunque se presenta con frecuencia, es un problema de difícil identificación [20]. Si bien las manifestaciones del maltrato físico pueden ser observables, otras formas no presentan características tan notorias.

Entre las dificultades para la detección de los casos están: la poca información acerca del problema; la incredulidad; los pensamientos negativos y el desagrado hacia la vejez; el aislamiento de las personas mayores; el miedo a denunciar; la vergüenza; la dependencia, que obstaculiza informar el caso, y el desconocimiento de procedimientos para denunciar [6]. Entre las principales barreras para la identificación del maltrato, algunos autores destacan la falta de una definición consensuada sobre el problema $[36,53]$.

Con frecuencia, las personas mayores no sereconocen como víctimas de maltrato, por diferentes motivos, como temor a represalias o a ser institucionalizados en Centros de bienestar del adulto mayor; prefieren negar la realidad que les resulta difícil de aceptar. Además, los familiares o responsables del cuidado tienden a ocultar estos hechos [49].

Diferentes signos de alarma o manifestaciones que de modo indirecto puedan advertir que se ha presentado maltrato, son de gran ayuda, debido a la cantidad de casos en los cuales es la única señal de sospecha. Entre los signos de alarma más comunes se encuentran la malnutrición, fracturas, deshidratación, úlceras por presión, síndrome de caídas, lesiones en la piel, alteraciones del sueño, polifarmacia, inmovilidad y mala higiene corporal [24].

Las principales formas en las cuales se hace control o identificación de este problema es por medio de los reportes a departamentos de emergencia [54], servicios de protección [55-58], expedientes del departamento de familia [59], denuncias al sistema [60], o por medio de la evaluación médica y el registro de traumas en el cuerpo, que den indicios de maltrato físico [61,62]. Sin embargo, hay un alto subregistro, debido a la poca denuncia por parte de las víctimas; en este sentido, han sido desarrollados estudios con la finalidad de estimar la magnitud real de este problema $[63,64]$, los cuales utilizan diversas escalas de medición para mayor objetividad y comparabilidad de los resultados [65]. La dificultad con esto radica en la variabilidad de este problema entre las distintas culturas, lo que implica que escalas desarrolladas para poblaciones específicas requieran ser adaptadas para el uso en otros contextos. Es por esto por lo que lo recomendado es la construcción de instrumentos que tengan en cuenta la diversidad cultural de cada región, dado que esto es fundamental para comprender el fenómeno $[33,66]$. En la tabla 1 se muestran las escalas de medición más utilizadas a nivel mundial.

Tabla 1. Instrumentos diseñados para evaluar maltrato hacia personas mayores.

\begin{tabular}{ll}
\hline \multicolumn{1}{c}{ Instrumento } & \multicolumn{1}{c}{ Descripción } \\
\hline Revised Conflict Tactics Scales (CTS) $[48,67-70]$ & $\begin{array}{l}\text { Herramienta creada por Murray A. Straus en 1979, utilizada para } \\
\text { realizar investigación sobre violencia familiar }\end{array}$ \\
\hline Vulnerability to Abuse Screening Scale (VASS) [71,72] & $\begin{array}{l}\text { Instrumento autodiligenciable que busca identificar mujeres } \\
\text { adultas mayores con riesgo de sufrir situaciones de maltrato }\end{array}$ \\
\hline Hwalek-Sengstock Elder Abuse Screening Test (H-S/EAST) & $\begin{array}{l}\text { Instrumento para medir situaciones de abuso, características } \\
\text { de vulnerabilidad y situaciones que pudieran ser consideradas } \\
{[73,74]}\end{array}$ \\
\begin{tabular}{ll} 
como abusivas contra el adulto mayor \\
\hline Caregiver Abuse Screen (CASE) [75]
\end{tabular} & $\begin{array}{l}\text { Instrumento para cuidadores que consta de ocho ítems, que } \\
\text { están redactados para que el encuestado se sienta cómodo al } \\
\text { contestarlos }\end{array}$ \\
\hline
\end{tabular}




\begin{tabular}{ll}
\hline \multicolumn{1}{c}{ Instrumento } & \multicolumn{1}{c}{ Descripción } \\
\hline $\begin{array}{ll}\text { Índice completo de malos tratos de Sengstock-Hwalek } \\
\text { (SHCIEA) [76] }\end{array}$ & $\begin{array}{l}\text { Instrumento para evaluar maltrato físico, psicológico, } \\
\text { económico, negligencia física y psicológica y violación a los } \\
\text { derechos como persona. Es considerado una de las escalas } \\
\text { más completas. Su desventaja es el tiempo requerido para su } \\
\text { aplicación, ya que contiene gran número de preguntas }\end{array}$ \\
\hline Breve examen de malos tratos BASE [77] & $\begin{array}{l}\text { Cuestionario corto para determinar si la persona mayor recibe } \\
\text { o no los cuidados apropiados para su edad. Permite tener una } \\
\text { visión general sobre maltrato físico, psicosocial, económico y } \\
\text { negligencia }\end{array}$ \\
\hline Expanded IOA (E-IOA) [78] & $\begin{array}{l}\text { Instrumento conformado por una escala de cuatro pasos para } \\
\text { clasificar el riesgo de maltrato }\end{array}$ \\
\hline Validation of the Indicators of Abuse (IOA) Screen [79] & Cuestionario de 27 ítems que indican características mentales y \\
& psicosociales de personas mayores y sus cuidadores familiares \\
\hline
\end{tabular}

En Latinoamérica, para el año 2013 fue desarrollada una escala para detectar maltrato, específicamente para mexicanos y personas hispanohablantes, llamada "Escala geriátrica de maltrato" (Geriatric Mistreatment Scale, GMs) [80], la cual toma en cuenta los cinco tipos de maltrato más frecuentes (físico, psicológico, económico, sexual y negligencia). Para el 2017, fue creada otra escala adaptada social y culturalmente para adultos mayores mexicanos, para ser aplicada en un primer nivel de atención [81].

\section{Magnitud del problema}

Aunque en las últimas décadas se ha comenzado a considerar mundialmente a los adultos mayores como población susceptible de recibir malos tratos, este es un problema aún invisibilizado [21]. Se estima que solo se conoce una parte de la dimensión real, lo que se debe principalmente a las dificultades que hay para identificarlo y al poco reporte [28].

La diferencia en los tipos de estudios empleados para investigar el maltrato, la variabilidad en las poblaciones, medidas y definiciones pueden ser también causantes del desconocimiento de la magnitud real del problema [41].

El "Informe mundial sobre el envejecimiento y la salud", emitido por la Organización Mundial de la Salud en 2015 [82], resalta la necesidad de incluir cambios en la forma de concebir el aumento de la población adulta mayor y en las acciones que se llevan a cabo en torno al envejecimiento, las cuales deben desarrollarse desde un enfoque de derechos $\mathrm{y}$, además, ir encaminadas a garantizar bienestar y calidad de vida en la vejez.

Entre las estrategias que se han puesto en marcha para reducir las conductas violentas contra personas mayores, principalmente en países con altos ingresos económicos, se destacan la realización de campañas de sensibilización, la detección de posibles víctimas y agresores, y las intervenciones de apoyo a cuidadores [46].
El maltrato hacia la población mayor aumenta a medida que la población mundial envejece, afecta cerca de 141 millones de personas mayores en países de ingresos bajos y medios [29]. Se estima que, en el mundo, 1 de cada 10 adultos mayores que vive en sus casas es maltratado [5], pero solo se reporta 1 de cada 24 casos [4]. En países desarrollados, la prevalencia estimada de maltrato va del 4 al $6 \%$ [83]. Una revisión sistemática, publicada en 2008, encontró que la prevalencia de abuso en la vejez variaba entre 3,2 y 27,5\% [41].

Para el año 2017, Yon et al. efectuaron una revisión sistemática donde encontraron que este problema se ha mantenido prevalente, con tendencia al aumento; dicha revisión reportó un $16 \%$ de personas adultas mayores maltratadas en 28 países. De acuerdo con el tipo de maltrato, el más frecuente es el psicológico, con un $11,6 \%$, seguido del financiero $(6,8 \%)$, negligencia $(4,2 \%)$, físico $(2,6 \%)$ y sexual $(0,9 \%)$ [29].

En Europa se estima que aproximadamente entre el 1 y el $3 \%$ de los adultos mayores que viven con sus familiares son maltratados [84]. Específicamente en España, la prevalencia del maltrato es de 29,3\% [85].

También se han reportado prevalencias del problema en otros países, como en Reino Unido, de un $2,6 \%$ [86]; Israel, de 18,4\% [87], y en India, de $14 \%$. En este último, se reporta que el maltrato verbal es el más frecuente, seguido del económico [35].

En América del norte, las prevalencias son variables. En Canadá, para el año 2013, se reportó una prevalencia de $43 \%$, siendo más frecuente el psicológico [88]. En Estados Unidos, en el 2014 fue publicado un estudio que encontró una prevalencia para maltrato verbal del $38 \%$ [68]. Específicamente, en Nueva York han sido reportadas prevalencias de maltrato emocional del 1,9\%; físico, de 1,8\%, y abandono, 1,8\% [67]. En México, para el año 2005, la prevalencia de maltrato psicológico era de aproximadamente $7 \%$; físico, del 3,4\%; económico, del 2,2 \%, y sexual, del 0,6 \% [89]. Para el año 2013, otro estudio realizado en México en población mayor 
no institucionalizada reportó prevalencias que oscilaban entre 8,1 y $10,3 \%$ [80].

En la Segunda Conferencia Regional Intergubernamental sobre Envejecimiento en América Latina y el Caribe: hacia una sociedad para todas las edades y de protección social basada en derechos, realizada del 4 al 6 de diciembre de 2007 en Brasilia (Brasil), el maltrato hacia las personas mayores fue presentado como un problema que tiene implicaciones directas en la práctica y garantía de los derechos humanos, por lo que se reafirmó el compromiso por aumentar los esfuerzos para proteger los derechos y las libertades de las personas de edad avanzada, para erradicar todas las formas de discriminación y abuso [90].

En Colombia, la "Política Nacional de Envejecimiento y Vejez (2007-2019)" establece la necesidad de crear condiciones adecuadas para garantizar una vida saludable y digna a las personas mayores, incluyendo los cuidados adecuados, el acceso a vivienda, alimentación y seguridad social. Además, en esta política se incluye la protección social integral del adulto mayor, donde se resalta la necesidad de generar entornos seguros y saludables para los mayores [2].

El informe de violencia intrafamiliar del Instituto de Medicina Legal y Ciencias Forenses del año 2012 [45] muestra el aumento de este problema en los últimos años, con un ascenso desde el año 2007 (965 casos), con su mayor pico en el año 2010, donde se reportaron 1631 casos de violencia contra el adulto mayor. Para el año 2012, los casos de maltrato se presentaron en mayor medida en mujeres (53\%). Respecto al rango de edad, se produjo principalmente en adultos mayores de 60 a 69 años. Los principales responsables de las agresiones fueron los hijos.

La encuesta SABE Colombia, realizada en el año 2015 [12], encontró una prevalencia de maltrato al adulto mayor de $12,3 \%$, siendo más frecuente en mujeres, para todos los tipos de maltrato, y en menores de 70 años. También fue más frecuente en personas separadas y de bajos ingresos económicos.

Dicho estudio halló que los adultos con edades entre 65 y 69 años reportaron, principalmente, maltrato psicológico $(14,0 \%)$ y físico $(3,6 \%)$. Los adultos de piel clara reportaron, en especial, maltrato psicológico, mientras que los de piel oscura indicaron más maltrato físico, financiero y sexual [12].

La tipología más frecuente de maltrato en el país fue el psicológico, con un $12,3 \%$, seguido del físico $(3,4 \%)$, financiero $(1,5 \%)$ y sexual $(0,3 \%)$.
El maltrato psicológico fue reportado principalmente en personas de estratos 1 y $2 ;^{\dagger}$ en las personas de estrato 1 se identificó mayor maltrato físico y sexual, mientras que en los estratos 5 y 6 se encontró mayor maltrato económico.

En el área urbana fue más frecuente el psicológico, mientras que en el área rural el porcentaje fue alto para los demás tipos de maltrato. La negligencia fue principalmente reportada en mujeres $(9,6 \%)$ y en el área rural $(11,3 \%)$ [12].

Para el año 2016, de acuerdo con un estudio realizado en adultos mayores en tres ciudades de Colombia: Medellín, Barranquilla y Pasto, se encontró que la prevalencia de maltrato en Pasto era del 13,0 \%, siendo más frecuente el maltrato psicológico. Dicho estudio reportó que los principales factores asociados a este problema fueron la dependencia "para realizar actividades básicas de la vida diaria, los malos tratos entre los miembros de la familia y el escaso apoyo social" [91].

En Bogotá [63] se realizó un estudio con los datos de la encuesta SABE 2012, en el cual se analizó la asociación entre historia de maltrato y caídas en adultos mayores. Dicho estudio encontró que un 33,8 \% de los adultos mayores había sufrido alguna de las formas de maltrato, y el $13,7 \%$, más de un tipo de maltrato. La prevalencia de maltrato psicológico fue de 26,9 \%; físico, 19,4\%, y sexual, 2,8 \%. Este estudio concluyó que los adultos mayores que alguna vez habían sido maltratados, presentaban altas probabilidades de caídas.

En Santa Marta, un estudio realizado entre 2005 y 2009, acerca de estilos de vida de los adultos mayores, indagó sobre el maltrato, encontrando que el tipo más frecuente fue el económico, con un $38 \%$, seguido del psicológico, con un $7 \%$, y el de la negligencia, con un $3 \%$ [92].

En Antioquia, un estudio realizado en el año 2012, sobre la situación de salud y condiciones de vida del adulto mayor [10], halló que el maltrato más reportado fue el psicológico, con un $5 \%$. Este tipo de maltrato fue más frecuente en mujeres $(5,4 \%)$ que en hombres (4,0 \%); los centenarios (mayores de 100 años) tuvieron prevalencias más altas que los jóvenes (60-74 años); se presentó principalmente en personas solteras y sin ningún nivel educativo. Finalmente, dicho estudio encontró que Medellín fue la ciudad con mayor prevalencia de maltrato psicológico $(6,3 \%)$ [11].

$\dagger$ En Colombia, las viviendas y los predios rurales son clasificadas en estratos socioeconómicos que van del estrato más bajo (estrato 1), que alberga personas con menores recursos, hasta el más alto, que es 6, en el cual se encuentran las personas con mayores recursos económicos. 


\section{Conclusiones}

El envejecimiento poblacional trae consigo grandes retos, tanto para los gobiernos, como para las familias y la comunidad, entre los cuales se destaca superar la discriminación en razón de la edad, proporcionar calidad de vida a los adultos mayores, contrarrestar el maltrato contra esta población y brindar una adecuada atención y cuidado. El maltrato al adulto mayor es ejercido por una sociedad en la que prevalece una imagen negativa y estereotipada de la vejez. Este problema refleja la necesidad de concientizar a la población acerca del respeto por el envejecimiento y de generar redes de apoyo para garantizar bienestar en esta etapa.

Dada la carga cultural que tiene este problema, la definición de maltrato puede variar en cada sociedad, $\mathrm{y}$ un primer paso para la adecuada evaluación y disminución de estos hechos violentos es partir de una definición consensuada, que sea reflejo de las características culturales, sociales y familiares. A partir de una conceptualización en contexto del problema, se pueden desarrollar y poner en práctica actividades en las que se incluya la familia, para la reducción del maltrato y la minimización del impacto negativo que tiene este sobre las víctimas.

La alta subestimación que hay de la magnitud de este problema no solo se apoya en la falta de herramientas adecuadas, sino también en la falta de educación para realizar una oportuna y adecuada identificación. En Colombia, es poco lo que se conoce acerca de la magnitud y las características que facilitan o desencadenan este tipo de actos violentos al interior de las familias. Hace falta la realización de investigación que permita orientar planes y programas que busquen la visibilización de este problema en la sociedad, pero también la disminución de casos y la atención oportuna para evitar las repercusiones negativas que trae sobre la víctima. Es necesario profundizar en conocer el papel protector que puede desempeñar la cohesión y el buen funcionamiento familiar, además del fortalecimiento de las redes sociales de los adultos mayores en el país.

\section{Conflictos de interés}

Se declara que para la elaboración del ensayo no hubo conflictos de interés de los autores.

\section{Financiación}

Se declara que para la elaboración del ensayo, Maite Catalina Agudelo Cifuentes tuvo financiación de Colciencias (Becas Doctorados Nacionales, convocatoria 272/2015).

\section{Declaración de responsabilidad}

Los puntos de vista expresados en este artículo son responsabilidad de los autores y no de la Institución donde trabajan o de la entidad financiadora.

\section{Declaración de autoría}

Maite Catalina Agudelo Cifuentes formuló la pregunta de investigación, realizó estrategia de búsqueda de literatura, lectura de artículos, evaluación de artículos y redactó el manuscrito. Cardona Arango, Segura Cardona y Restrepo Ochoa participaron en la lectura y la evaluación de artículos, en la revisión y la aprobación del manuscrito.

\section{Referencias}

1. Organización Mundial de la Salud. Envejecimiento [internet]. s. f. [citado 2018 mar. 27]. Disponible en: http://www.who.int/topics/ ageing/es/.

2. Ministerio de la Protección Social. Política Nacional de Envejecimiento y Vejez (2007-2019) [internet]. 2007 [citado 2018 oct. 12]. Disponible en: https://www.minsalud.gov.co/ Documentos\%20y\%20Publicaciones/POL\%C3\%8DTICA\%20 NACIONAL \%20DE\%20ENVEJECIMIENTO\%20Y\%20 VEJEZ.pdf.

3. Lemus $\mathrm{S}$ de, Expósito F. Nuevos retos para la psicología social: edadismo y perspectiva de género. Pensam Psicológico. 2005;1(4):33-51.

4. Organización Mundial de la Salud (OMS). Aumenta el maltrato a las personas de edad: según la OMS, afecta a uno de cada seis ancianos [internet]. 2017. [citado 2018 mar. 27]. Disponible en https://www.who.int/es/news-room/detail/14-06-2017-abuse-ofolder-people-on-the-rise-1-in-6-affected.

5. Centers for Disease Control and Prevention. Elder Abuse Prevention [internet]. 2016 [citado 2018 oct. 9]. Disponible en: https://www.cdc.gov/features/elderabuse/.

6. Bover A, Moreno M, Mota S, et al. El maltrato a los ancianos en el domicilio. Situación actual y posibles estrategias de intervención. Aten Primaria. 2003;32(9):541-51.

7. Departamento Administrativo Nacional de Estadística. Proyecciones nacionales y departamentales de población 20052020. Estudios Postcensales No. 7. Bogotá: Departamento Administrativo Nacional de Estadística.

8. Dulcey E, Arrubla D, Sanabria P. Envejecimiento y vejez en Colombia. Estudio a profundidad [informe de investigación] [Internet]. 2013 [citado 2020 ene. 29]. Disponible en: https:// fiapam.org/wp-content/uploads/2014/03/3-ENVEJECIMIENTOY-VEJEZ-EN-COLOMBIA.pdf

9. Colombia, Ministerio de Salud y Protección Social, Oficina de Promoción Social. Envejecimiento demográfico. Colombia 1951-2020. Dinámica demográfica y estructuras poblacionales [internet]. 2013 [citado 2017 ago. 8] Disponible en: https://www. minsalud.gov.co/sites/rid/Lists/BibliotecaDigital/RIDE/DE/PS/ Envejecimiento-demografico-Colombia-1951-2020.pdf.

10. Cardona D, Segura A, Garzón M. Situación de salud y condiciones de vida del adulto mayor. Departamento de Antioquia, 2012. Medellín: Universidad CES, Gobernación de Antioquia; 2013. 
11. Cano S, Garzón M, Segura A, et al. Maltrato psicológico en los adultos mayores del departamento de Antioquia, 2012. Rev Fac Nac Salud Pública. 2014;21(Supl. 1):S99-S106.

12. Colombia, Ministerio de Salud y Protección Social. Ministerio de Salud y Protección Social. SABE Colombia. Encuesta Nacional de Salud, Bienestar y Envejecimiento; 2015.

13. Burston G. Letter: Granny-battering. Br Med J. 1975;3:592. DOI: http://dx.doi.org/10.1136/bmj.3.5983.592-a.

14. Johnson, T.F. Critical issues in the definition of Elder mistreatment En: K. Pillemer, R. Wolf, editores. Elder abuse: Conflict in the Family. Dover, MA: Auburn House Publishing Company; 1986. pp. 167-96.

15. Hudson M. Elder mistreatment: A taxonomy with definitions by Delphi. J Elder Abuse Negl. 1991;3(2):1-20. DOI: https://doi org/10.1300/J084v03n02_01.

16. Kessel H, Marín N, Maturana N. Primera Conferencia Nacional de Consenso sobre el Anciano Maltratado. Rev Esp Geriatr Gerontol. 1996;31:367-72.

17. Álvarez C. Crisis intergeneracional y anomia cultural, la marginación y maltrato al anciano. En: Memorias del Foro Nacional sobre Tercera edad "Hacia una política de participación de los viejos". Procuraduría delegada para la defensa del menor y la familia. Bogotá; 1997.

18. Organización Panamericana de la Salud. Informe mundial sobre la violencia y la salud. Resumen [internet]. 2002 [citado 2016 feb. 24]. Disponible en: http://www.who.int/violence_injury_ prevention/violence/world_report/es/summary_es.pdf.

19. Organización Mundial de la Salud. Declaración de Toronto para la prevención global del maltrato de las personas mayores [internet]. 2003 [citado 2016 feb. 24]. Disponible en: http://www. who.int/ageing/projects/elder_abuse/alc_toronto_declaration es.pdf?ua $=1$.

20. Muñoz J. Personas mayores y malos tratos. Madrid: Pirámide; 2004.

21. De Paúl J, Larrión JL. El maltrato a los mayores. Algunas cuestiones generales. En: Cuesta J, editor. El maltrato de personas mayores. Detección y prevención desde un prisma criminológico interdisciplinar. Donostia, Gipuzkoa: Hurkoa Fundazioa, Instituto Vasco de Criminología; 2006. pp. 11-15.

22. Iborra I. Maltrato de personas mayores en la familia en España. Valencia: Fundación de la Comunitat Valenciana para el Estudio de la Violencia; 2008.

23. Gobierno de Chile. Servicio Nacional del Adulto Mayor. Guía Prevención del maltrato a las personas mayores [internet]. 2012 [citado 2017 ago. 20]. Disponible en http://www.senama.gob. cl/storage/docs/Guia-Prevencion-del-maltrato-a-las-personasmayores.pdf.

24. México, Gobierno Federal. Guía de práctica clínica. Detección y manejo del maltrato en el adulto mayor. México: Instituto Mexicano del Seguro Social; 2013.

25. Giraldo-Rodríguez L, Rosas-Carrasco O, Mino-León D Abuse in Mexican older adults with long-term disability: National prevalence and associated factors. J Am Geriatr Soc. 2015;63:1594-600. DOI: https://doi.org/10.1111/jgs.13552.

26. Compton SA, Flanagan P, Gregg W. Elder abuse in people with dementia in Northern Ireland: Prevalence and predictors in cases referred to a psychiatry of old age service. Int J Geriatr Psychiatry. 1997;12(6):632-5.

27. Servicio Nacional del Adulto Mayor. Definición y tipificación del maltrato al adulto mayor, en Chile [Internet]. s. f [citado 2020 ene.
29]. Disponible en: http://www.senama.gob.cl/storage/docs/Defitipif-maltrato-adulto-mayor-Chile-2005.pdf

28. Morena B. Maltrato psicológico. En: Cuesta J, editor. El maltrato de personas mayores. Detección y prevención desde un prisma criminológico interdisciplinar. Donostia, Gipuzkoa: Hurkoa Fundazioa, Instituto Vasco de Criminología; 2006. pp. 23-38.

29. Yon Y, Mikton CR, Gassoumis ZD, et al. Elder abuse prevalence in community settings: A systematic review and meta-analysis. Lancet Glob Health. 2017;5(2):e147-56. DOI: https://doi. org/10.1016/S2214-109X(17)30006-2.

30. Organización Mundial de la Salud. Maltrato de las personas mayores [Internet]. 2018. [citado 2019 jun. 13]. Disponible en https:/www.who.int/es/news-room/fact-sheets/detail/elder-abuse

31. Iborra I. Factores de riesgo del maltrato de personas mayores en la familia en población española. Zerbitzuan. 2009;(45):49-57.

32. Acierno R, Hernandez MA, Amstadter AB, et al. Prevalence and correlates of emotional, physical, sexual, and financial abuse and potential neglect in the United States: The national elder mistreatment study. Am J Public Health. 2010;100(2):292-7. DOI: https://doi.org/10.2105/AJPH.2009.163089.

33. Melchiorre MG, Rosa MD, Lamura G, et al. Abuse of older men in seven European countries: A multilevel approach in the framework of an ecological model. PLOS ONE. 2016;11(1):e0146425. DOI: https://doi.org/10.1371/journal.pone.0146425.

34. Pérez-Rojo G, Penhale B. Maltrato de las personas mayores: situación actual en el Reino Unido. Rev. Esp. Geri. y Geront. 2006;41(5):289-96. DOI: 10.1016/S0211-139X(06)72975-4.

35. Chokkanathan S, Lee AE. Elder mistreatment in urban India: A community based study. J Elder Abuse Negl. 2005;17(2):45-61. DOI: https://doi.org/10.1300/J084v17n02_03.

36. Penhale B. Older women, domestic violence, and elder abuse: A review of commonalities, differences, and shared approaches. J Elder Abuse Negl. 2003;15(3-4):163-83. DOI: https://doi. org/10.1300/J084v15n03_10.

37. Bazo M. Incidencia y prevalencia del maltrato hacia los mayores. En: Cuesta J, editor. El maltrato de personas mayores. Detección y prevención desde un prisma criminológico interdisciplinar. Donostia, Gipuzkoa: Hurkoa Fundazioa, Instituto Vasco de Criminología; 2006. pp. 39-60.

38. Homer A, Gilleard C. Abuse of elderly people by their carers. BMJ. 1990;301(6765):1359-62. DOI: https://doi.org/10.1136/ bmj.301.6765.1359.

39. Organización Mundial de la Salud. Maltrato de ancianos y alcohol. Nota descriptiva [internet]. 2006 [citado 2017 ago. 13]. Disponible en: http://www.who.int/violence_injury_prevention/ violence/world_report/factsheets/fs_elder_es.pdf.

40. Coyne A, Reichman W, Berbig L. The relationship between dementia and elder abuse. Am J Psychiatry. 1993;150(4): 643-6. DOI: https://doi.org/10.1176/ajp.150.4.643.

41. Cooper C, Selwood A, Livingston G. The prevalence of elder abuse and neglect: A systematic review. Age Ageing. 2008;37(2):15160. DOI: https://doi.org/10.1093/ageing/afm194.

42. Echeburúa E. Manual de violencia familiar. Madrid: Siglo XXI; 1998.

43. Abello R, Madariaga C. Las redes sociales ¿Para Qué? Psicolía desde El Caribe. 1999;(2-3):116-35.

44. Sierra O, Madariaga C, Abello R. Relación entre la pertenencia a redes sociales y dinámica familiar de mujeres trabajadoras en condiciones de pobreza. Psicología desde El Caribe. 1998;(1):94-113. 
45. Instituto Nacional de Medicina Legal y Ciencias Forenses, Subdirección de Servicios Forenses, Grupo Centro de Referencia Nacional sobre Violencia. Violencia al adulto mayor en el contexto intrafamiliar. Boletín; 2012.

46. Organización Mundial de la Salud. Maltrato de las personas mayores. Centro de prensa. [internet]. 2018 [citado 2020 enero 29]. Disponible en: http://www.who.int/mediacentre/factsheets/ fs $357 /$ es/.

47. Comisión Económica Para América Latina y el Caribe. Estrategia regional de implementación para América Latina y el Caribe del Plan de Acción Internacional de Madrid sobre el Envejecimiento. Santiago, Chile [internet]. 2004 [citado 2017 ago. 20]. Disponible en: https://www.cepal.org/es/publicaciones/2786-estrategiaregional-implementacion-america-latina-caribe-plan-accion.

48. De Donder L, Lang G, Penhale B, et al. Item non-response when measuring elder abuse: Influence of methodological choices. Eur J Public Health. 2013;23(6):1021-6. DOI: https://doi.org/10.1093/ eurpub/cks172.

49. Fernández-Alonso MC, Herrero-Velázquez S. Maltrato en el anciano. Posibilidades de intervención desde la atención primaria (I). Aten Primaria. 2006;37(1):56-59. DOI: https://doi. org/10.1157/13083927.

50. Dong X, Simon MA. Vulnerability risk index profile for elder abuse in community-dwelling population. J Am Geriatr Soc. 2014;62(1):10-15.

51. Lachs MS, Williams CS, O'Brien S, et al. The mortality of elder mistreatment. JAMA 1998;280(5):428-32. DOI: https://doi. org/10.1001/jama.280.5.428.

52. Rovi S, Chen P-H, Vega M, et al. Mapping the elder mistreatment iceberg: U.S. hospitalizations with elder abuse and neglect diagnoses. J Elder Abuse Negl. 2009;21(4):346-59. DOI: https:// doi.org/10.1080/08946560903005109.

53. House of Commons Health Select Committee. Elder abuse. Second Report of Session 2003-04. Vol. 1 [internet]. 2004 [citado 2017 ago. 14]. Disponible en: https://publications.parliament.uk/ $\mathrm{pa} / \mathrm{cm} 200304 / \mathrm{cmselect} / \mathrm{cmhealth} / 111 / 111 . p d f$

54. Evans CS, Hunold KM, Rosen T, et al. Diagnosis of elder abuse in U.S. emergency departments. J Am Geriatr Soc. 2017;65(1):91-7. DOI: https://doi.org/10.1111/jgs.14480.

55. Burnes D, Rizzo VM, Gorroochurn P, et al. Understanding Service utilization in cases of elder abuse to inform best practices. J Appl Gerontol. 2016;35(10):1036-57. DOI: https://doi. org/10.1177/0733464814563609.

56. Irigaray TQ, Esteves CS, Pacheco JTB, et al. Maus-tratos contra idosos em Porto Alegre, Rio Grande do Sul: um estudo documental. Estud. Psicol. (Campinas). 2016;33(3):543-51. DOI: http://dx.doi.org/10.1590/1982-02752016000300017.

57. Roepke-Buehler SK, Dong X. Perceived stress and elder abuse: A population-based study of adult protective services cases in Chicago. J Am Geriatr Soc. 2015;63(9):1820-8. DOI: http:// dx.doi.org/10.1111/jgs.13613.

58. Hwalek MA, Neale AV, Goodrich CS, et al. The association of elder abuse and substance abuse in the Illinois Elder Abuse System. Gerontologist. 1996;36(5):694-700. DOI: https://doi. org/10.1093/geront/36.5.694.

59. Sánchez Salgado CD. Características del abuso y maltrato de personas ancianas en Puerto Rico. P R Health Sci J. 2007;26(1):35-41.

60. Neale AV, Hwalek MA, Goodrich CS, et al. The Illinois elder abuse system: Program description and administrative findings. Gerontologist. 1996;36(4):502-11.
61. Ziminski CE, Wiglesworth A, Austin R, et al. Injury patterns and causal mechanisms of bruising in physical elder abuse. $\mathrm{J}$ Forensic Nurs. 2013;9(2):84-91. DOI: https://doi.org/10.1097/ JFN.0b013e31827d51d0.

62. Wiglesworth A, Austin R, Corona M, et al. Bruising as a marker of physical elder abuse. J Am Geriatr Soc. 2009;57(7):1191-6. DOI: https://doi.org/10.1111/j.1532-5415.2009.02330.x.

63. Reyes-Ortiz CA, Ocampo-Chaparro JM, Campo-Arias A, et al. Association between history of abuse and falling in older adults. Journal of the American Geriatrics Society. 2018;66(8):1603-7. DOI: https://doi.org/10.1111/jgs.15432.

64. Williams JH, Davis M, Acierno R. Global prevalence of elder abuse in the community. En: Dong X, editor. Elder Abuse: Research, Practice and Policy. Cham, Switzerland: Springer International Company AG.; 2017. pp. 45-65.

65. Gallione C, Dal A, Cristina FV, et al. Screening tools for identification of elder abuse: A systematic review. J Clin Nurs. 2017;26(15-16):2154-76. DOI: https://doi.org/10.1111/ jocn. 13721 .

66. Dong XQ. Culture diversity and elder abuse: Implications for research, education and policy. Generations. 2012;36(3):40-42.

67. Burnes D, Pillemer K, Caccamise PL, et al. Prevalence of and risk factors for elder abuse and neglect in the community: A population-based study. J Am Geriatr Soc. 2015;63(9):1906-12. DOI: https://doi.org/10.1111/jgs.13601

68. Fulmer T, Rodgers RF, Pelger A. Verbal mistreatment of the elderly. J Elder Abuse Negl. 2014;26(4):351-64. DOI: https://doi. org/10.1080/08946566.2013.801817.

69. Pillemer K, Finkelhor D. The prevalence of elder abuse: A random sample survey. Gerontologist. 1988;28(1):51-7. DOI: https://doi. org/10.1093/geront/28.1.51

70. Reay AM, Browne KD. Risk factor characteristics in carers who physically abuse or neglect their elderly dependants. Aging Ment Health. 2001;5(1):56-62.

71. Schofield MJ, Mishra GD. Validity of self-report screening scale for elder abuse: Women's health Australia study. The Gerontologist. 2003;43(1):110-20. DOI: https://doi.org/10.1093/ geront/43.1.110.

72. Schofield MJ, Reynolds R, Mishra GD, et al. Screening for vulnerability to abuse among older women: Women's health Australia study. J Appl Gerontol 2002;21(1):24-39. DOI: https:// doi.org/10.1177/0733464802021001002.

73. Edirisinghe PAS, Paranitharn P, Perera WNS, et al. Elder abuse among outpatient department attendees in a tertiary care hospital in Sri Lanka. Ceylon Med J. 2014;59(3):84-9. DOI: http://doi. org/10.4038/cmj.v59i3.6527.

74. Saikia AM, Mahanta N, Mahanta A, Deka AJ, Kakati A. Prevalence and risk factors of abuse among community dwelling elderly of Guwahati City, Assam. Indian J Community Med. 2015;40(4):279-81. DOI: https://doi.org/10.4103/09700218.164406

75. Reis M, Nahmiash D. Validation of the caregiver abuse screen (CASE). Can J Aging 1995;14(Supl. 2):45-60. DOI: https://doi. org/10.1017/S0714980800005584.

76. Sengstock M, Hwalek M. A critical analysis of measures for the identification of physical abuse and neglect in the elderly. Home Health Care Quarterly. 1986;6(4):27-39. DOI: https://doi. org/10.1300/J027v06n04_04.

77. National Initiative for the Care of the Elderly NICE. BASE Brief Abuse Screen for the Elderly.[Internet] 2011 [citado 2020 
ene. 28]. Disponible en: http://www.nicenet.ca/files/U_of_T Nice_180318_BASE_Tool_Sep11.pdf.

78. Cohen M, Halevi S, Gagin R, et al. Development of a screening tool for identifying elderly people at risk of abuse by their caregivers. J Aging Health. 2006;18(5):660-85. DOI: https://doi. org $/ 10.1177 \% 2 \mathrm{~F} 0898264306293257$.

79. Reis, M., y Nahmiash, D. (). Validation of the indicators of abuse (IOA) screen. The Gerontologist. 1998;38(4):471-80. DOI: https://doi.org/10.1093/geront/38.4.471

80. Giraldo LG, Rosas O. Development and psychometric properties of the Geriatric Mistreatment Scale. Geriatr Gerontol Int. 2013;13(2):466-74. DOI: https://doi.org/10.1111/j.14470594.2012.00894.x.

81. Ruelas-González MG, Pelcastre-Villafuerte BE, MonterrubioFlores E, et al. Development and validation of a Screening Questionnaire of Family Mistreatment against Older Adults for use in primary care settings in Mexico. Health Soc Care Community. 2018;26(1):102-12. DOI: https://doi.org/10.1111/ hsc. 12466 .

82. Organización Mundial de la Salud. Informe mundial sobre el envejecimiento y la salud [internet]. 2015 [citado 2016 mar. 2]. Disponible en: https://apps.who.int/iris/bitstream/ handle/10665/186466/9789240694873_spa.pdf;jsessionid=155E 9FD3570DE8FE0F676DFA7BE98370? sequence $=1$.

83. Organización Mundial de la Salud. Datos interesantes acerca del envejecimiento. Envejecimiento y ciclo de vida [internet]; s. f. [citado 2018 mar. 3]. Disponible en: http://www.who.int/ageing/ about/facts/es/.

84. Hydle I. Violence against the elderly in western Europe. Treatment and preventive measures in the health and social service fields. J Elder Abuse Negl. 1989;1(3):75-87. DOI: https://doi.org/10.1300/ J084v01n03_07.
85. Garre-Olmo J, Planas-Pujol X, López-Pousa S, et al. Prevalence and risk factors of suspected elder abuse subtypes in people aged 75 and older. J Am Geriatr Soc. 2009;57(5):815-22. DOI: https:// doi.org/10.1111/j.1532-5415.2009.02221.x.

86. Biggs S, Manthorpe J, Tinker A, Doyle M, Erens B. Mistreatment of older people in the United Kingdom: Findings from the first national prevalence study. J Elder Abuse Negl. 2009;21(1):1-14. DOI: https://doi.org/10.1080/08946560802571870.

87. Lowenstein A, Eisikovits Z, Band-Winterstein T. Enosh G. Is Elder abuse and neglect a social phenomenon? Data from the First National Prevalence Survey in Israel. J Elder Abuse Negl. 2009; 21(3): 253-77. DOI: https://doi.org/10.1080/08946560902997629.

88. McDonald L, Thomas C. Elder abuse through a life course lens. Int Psychogeriatr. 2013;25(8):1235-43. DOI: https://doi.org/10.1017/ S104161021300015X.

89. Gómez Ricárdez LA, Rodríguez Abrego G, Krug Llamas E. Prevalencia y factores asociados a violencia familiar en adultos mayores de Ocozocoautla (Chiapas, México). Rev Esp Geriatr Gerontol. 2007;42(1):27-34. DOI: https://doi.org/10.1016/S0211139X(07)73518-7.

90. Comisión Económica para América Latina y El Caribe. Segunda Conferencia Regional Intergubernamental sobre Envejecimiento en América Latina y el Caribe: hacia una sociedad para todas las edades y de protección social basada en derechos. Brasilia, Brasil; 2007.

91. Agudelo-Cifuentes M.C, Cardona D, Segura A, et al. Características sociales y familiares asociadas al maltrato al adulto mayor de Pasto, Colombia 2016. Rev CES Psico. 2019;12(1):3242. DOI: http://dx.doi.org/10.21615/cesp.12.1.3.

92. Monroy CM. Estilo de vida. Adultos mayores de Santa Marta. Memorias. 2010;8(13):20-29. 